\title{
Correction to: Machine learning the nuclear mass
}

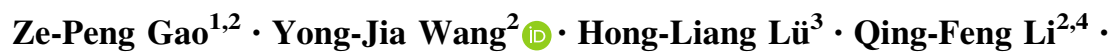 \\ Cai-Wan Shen ${ }^{2} \cdot$ Ling Liu ${ }^{1}$
}

Published online: 5 November 2021

(C) The Author(s), under exclusive licence to China Science Publishing \& Media Ltd. (Science Press), Shanghai Institute of Applied Physics, the Chinese Academy of Sciences, Chinese Nuclear Society 2021

\section{Correction to: NUCL SCI TECH}

https://doi.org/10.1007/s41365-021-00956-1

Following publication of the original article, Formula (2) is missing and Fig. 11, Fig. 9 are identical.

The original article has been corrected and the Publisher apologized to the authors and the readers for the inconvenience caused by this error.
The corrected version of Formula (2) and Fig. 11 are given below:

$$
E_{\mathrm{p}}= \begin{cases}\frac{d_{\mathrm{n}}}{N^{1 / 3}}+\frac{d_{\mathrm{p}}}{Z^{1 / 3}}+\frac{d_{\mathrm{np}}}{A^{2 / 3}}, & \text { for } Z \text { and } N \text { odd, } \\ \frac{d_{\mathrm{p}}}{Z^{1 / 3}}, & \text { for } Z \text { odd }, N \text { even, } \\ \frac{d_{\mathrm{n}}}{N^{1 / 3}}, & \text { for } Z \text { even, } N \text { odd, } \\ 0, & \text { for } Z \text { and } N \text { even. }\end{cases}
$$

The original article can be found online at https:// doi.org/10.1007/s41365-021-00956-1.

Yong-Jia Wang

wangyongjia@zjhu.edu.cn

$\bowtie$ Qing-Feng Li

liqf@zjhu.edu.cn

1 College of Physics Science and Technology, Shenyang Normal University, Shenyang 110034, China

2 School of Science, Huzhou University, Huzhou 313000, China

3 HiSilicon Research Department, Huawei Technologies Co., Ltd., Shenzhen 518000, China

4 Institute of Modern Physics, Chinese Academy of Science, Lanzhou 730000, China 


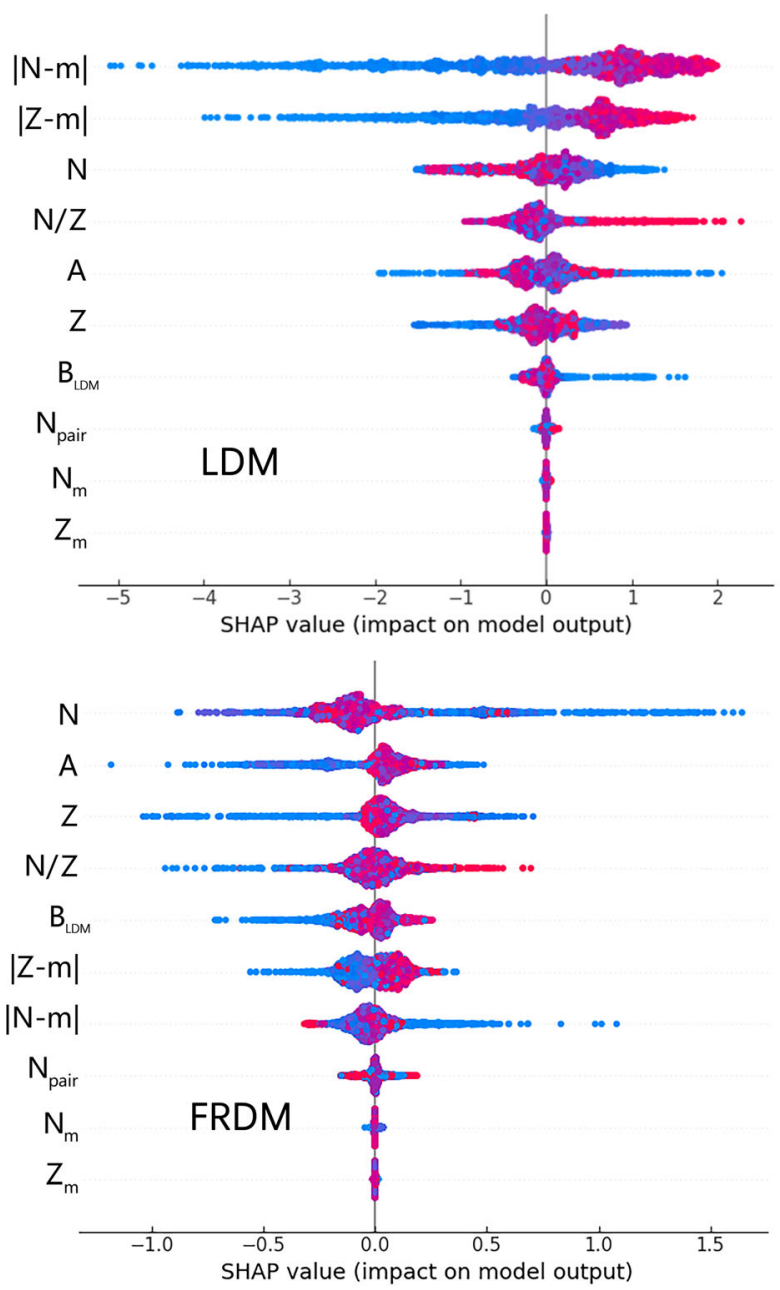

Fig. 11 (Color online) Importance ranking for the input features obtained with the SHAP package. Each row represents a feature, and the $x$-axis is the SHAP value, which shows the importance of a feature

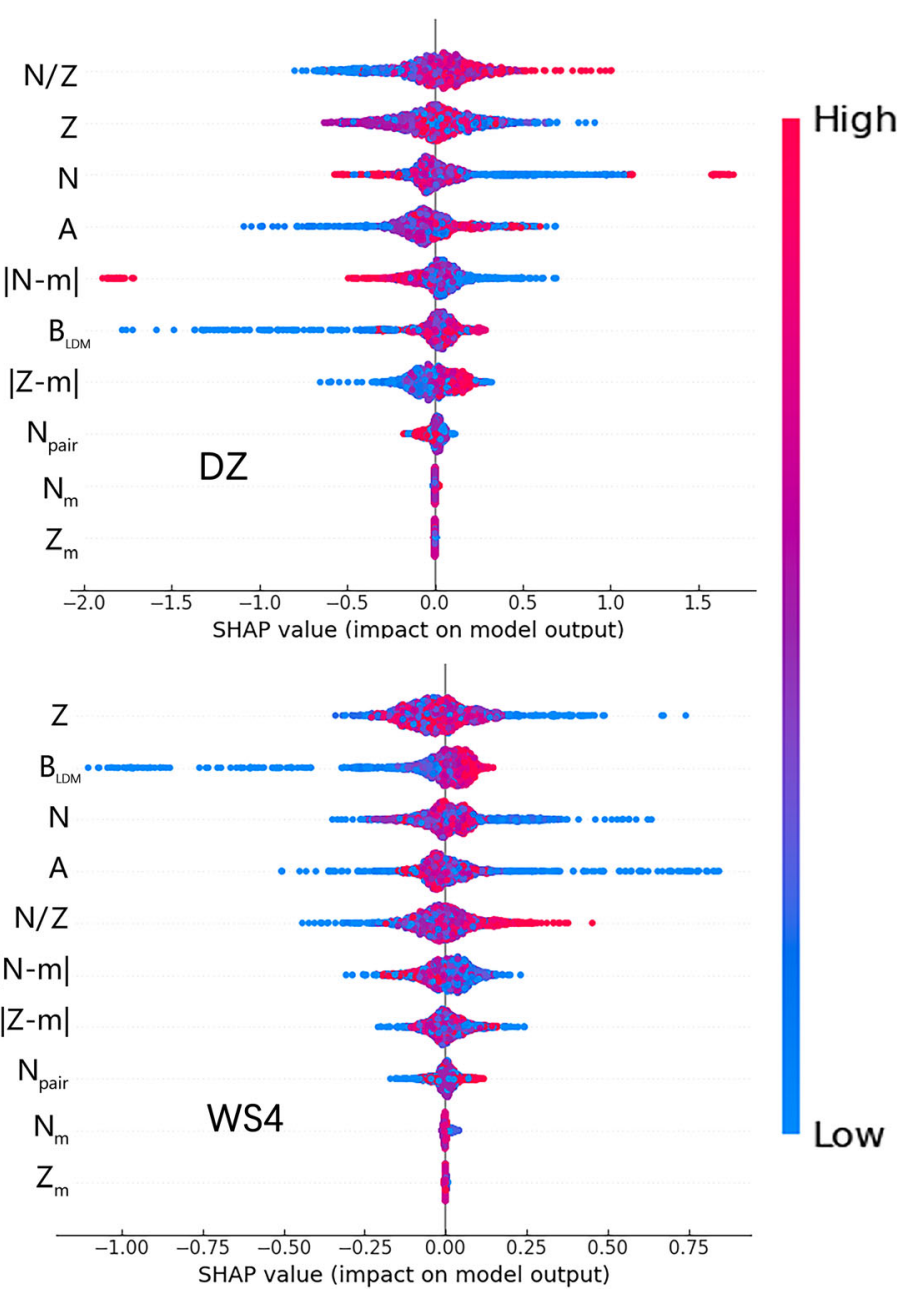

for a particular prediction. Each point represents a nucleus, and the color represents the feature value (with red being high and blue being low) 